\title{
Advanced Engineering Visualization with Standardized 3D Formats
}

\author{
Christian Emmer, Arnulf Fröhlich, and Josip Stjepandic \\ PROSTEP AG, Darmstadt, Germany \\ josip.stjepandic@opendesc.com
}

\begin{abstract}
When products are developed in 3D for the engineering domain, the data is initially stored in the native format of the used CAD software. If this $3 \mathrm{D}$ CAD data is to be made available to people who do not have this software, neutral 3D formats are needed. For visualization of product data in the engineering field - regardless of native CAD formats - are various 3D formats available. Among these are disclosed or standardized formats like PDF from Adobe, JT and also X3D, Collada and STEP. The choice of a format has many implications, including which options are available for using the data and what follow-up costs will result. This paper illustrates an overview of the state of development, the committees' activities as well as current examples from practice and gives outlook for future developments too. The record is completed by examples from the industrial practice in the automotive industry.
\end{abstract}

Keywords: Engineering Visualization, Interoperability, Data Exchange, JT Standard, Supply Chain, Engineering Collaboration.

\section{$1 \quad$ Introduction}

Although the CAD translation technology has gathered a high level of maturity and robustness, there is still a strong need for further development driven by crucial business processes like digital mock-up (DMU) which is established as core validation process in the past years. Furthermore the CAD models have already exceeded the level of complexity, where they are easy to handle with. Meanwhile various 3D visualization formats have entered the market on the broad front by combining the high information content with easy handling and excellent performance. Thus these formats can support many process chains from styling to product simulation, validation and production as well as downstream life cycle phases. By the extensive penetration of visualization formats in the market the old discussion was reopened, whether the unique format can fulfil the requirements of the engineering collaboration (internal and external). Finally the users need the recommendation too, which format meets their needs in the best way [1], [2]. Typical use cases for functional evaluation of such formats are the viewing of engineering data, the design in context, the data exchange between partners in the supply chain, the packaging and digital mock-up (DMU), the documentation and archiving, and use in the portable PLM document, i.e. use of 3D and additional information in domains related to engineering (Figure 1). 
After the rough evaluation of the technical specification of the visualization formats an overall comparison can be drawn [3] (Figure 2).

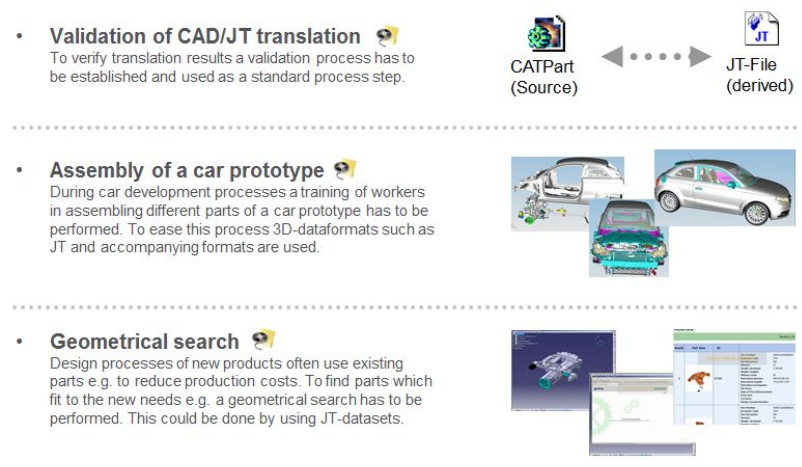

Fig. 1. Typical use cases of JT [1]



Fig. 2. Comparison of four visualization formats [3]

However in the automotive and aerospace industry JT from Siemens PLM is currently under heavy investigation to apply it in downstream processes after it was established as leading format for the engineering visualization in many global companies [4]. For instance, there is the demand to exchange exact geometry, product data information and additional attributes. Moreover JT is also a potential data format for long-term data retention. Consequently, there is high user interest in quality and robustness of the applications, the interfaces in particular. Thus the further explanation in this paper will be focused on JT although other formats (STEP, 3DPDF, 3DXML) are in the different stages of standardization procedure.

Open standards enable reduction of total costs of ownership and ensure independence from specific vendors and competition. With regard to visualization data exchange there is a strong support to use JT and STEP AP 242 (ISO 10303-242) as complementing standards for lightweight visualization format for $3 \mathrm{D}$ industrial data as well as product structure, meta, kinematic data [5].

The publication of the JT specification as an ISO Publicly Available Specification (PAS 14306) laid the foundation for establishing JT as a binding process format. The ProSTEP iViP Association and the VDA (German Association of the Automotive Industry) subsequently launched three coordinated JT-specific projects: the JT Workflow Forum, the JT Implementor Forum and the JT Application Benchmark. 


\section{Technical Background and Standardization}

JT is a binary format whose data model supports various representations of CAD geometry. The representations can be stored in a JT file individually or together [5] [6].

- BREP (Boundary Representation): Offers the highest level of representational precision. BREP data is compressed using different algorithms and stored without loss. In the current specification 9.5, two BREP representations are permitted: the traditional JT-BREP representation and XT-BREP, which is based on the Parasolid boundary representation and will be preferred in the further implementation of JT based software.

- Tessellated Geometry: A faceted representation of solids and surfaces. Different levels of detail (LOD) can be defined within a JT file. A low LOD means a lower level of precision but a smaller volume of data, while a very high LOD means an almost exact geometry but a large volume of data.

- ULP (Ultra-Lightweight Precise): The latest compression method is ULP. The ULP format enables a lightweight, semi-precise representation of the $3 \mathrm{D}$ geometry. The level of precision that ULP offers is significantly higher than for tessellated geometry while the file size is significantly smaller. The primary focus lies on providing high quality surface geometry that exhibits only minor deviations from the original BREP geometry.

JT version 8.1 has been initially published by the ISO as a publicly available specification (ISO PAS 14306). The ISO standardization process has been finished by endorsing the ISO standard (ISO 14306) in December 2012 for the recently published JT version 9.5, which has been expanded to include the specification for ULP and semantic product manufacturing information (PMI, product metadata) among other things.

Generating data in a neutral 3D format results in most use cases with a significant reduction in volume. In order to compare the file size of the 3D formats, fifteen test assemblies from different $\mathrm{CAD}$ systems were used to generate different $3 \mathrm{D}$ formats [7]. As a result the volume of data is determined more by data content then by the format itself.

The volume of exact BREP data after conversion to JT is approximately the same as after conversion to 3D PDF. The same applies to tessellated content, where the result for both formats is about the same as for 3D XML. In the case of simplified BREP data, the volume of data for both JT and 3D PDF are approximately the same. In the case of STEP data, use of an external compression algorithm achieves a marked reduction in size. As a consequence higher accuracy requires more storage space as a general rule. 


\section{Use Cases}

An evaluation of the 22 use cases defined by automotive manufacturers and their suppliers indicated a particularly high priority for four use cases, which are presented below in more detail by way of example [7] (Figure 3).

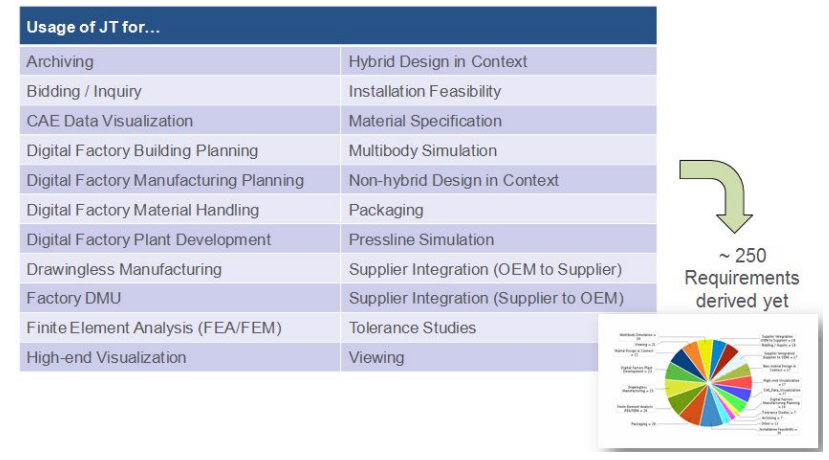

Fig. 3. Use cases for simplified formats [7]

Viewing: If the use of a CAD system is not desired, the visualization of engineering data using 3D viewers comes into play in a number of different situations: the presentation of product data, the representation of 3D models for information purposes (e.g. for a design review or marketing) and the realistic representation in virtual reality systems.

The use case can vary according to the concrete application context involved. While the simple viewing of the geometry is sufficient in many cases, in other cases metadata or high-performance viewing of huge assemblies plays a key role. The most important requirements are:

- Quality criteria for geometry accuracy must be fulfilled.

- Different level of details must be available.

- Detailed colour information must be available for entire part and single geometrical features.

- Metadata like attributes must be stored.

Packaging / DMU: In digital mock-up (DMU), the spatial properties of a product are examined and checked. This can involve checking the overall geometry with regard to dimensions and shape, interference checks, collision checks for assembly and disassembly, as well as design space checks (Figure 4).

For these purposes, the geometry, product structure and metadata are displayed and analyzed in a DMU application. The result of the DMU analysis is subsequently summarized and documented in a report. The most important requirements are:

- Use of models from different source systems (multi-CAD).

- High-quality examination of large assemblies. 
- Transferability of kinematics from the original model to the target model for dynamic DMU analysis.

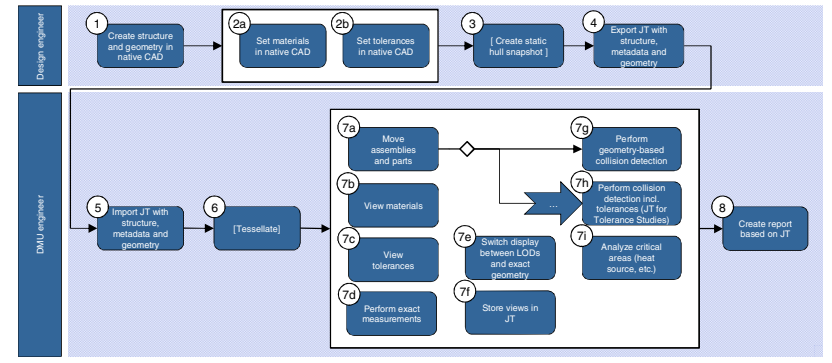

Fig. 4. Use case diagram "Packaging"

Design in Context: A typical used modeling method is the design in the context of the existing geometry. In that case existing CAD models (e.g. from previous design or from partners) are loaded into the CAD system. The design engineer then references new or existing JT-geometry to the loaded parts or assemblies. References from native CAD to JT are also in focus. These references can be between auxiliary geometry (point, axis, plane), geometrical elements such as edges, vertices or faces and exact geometrical references like curves. In the subsequent downstream processes it is required to use this assembled model as merged single unit. Hence the creation of technical drawings must be possible. The most important requirements are:

- Tessellated as well as exact B-Rep data must be stored.

- Links between JT and native data must be possible.

- Technical drawings must be derivable from hybrid models.

Partner Integration: The use case is divided into two sub sections. The aim of the "OEM to Supplier" use case is to send validated JT geometry with all needed metadata to the supplier, whereas the "Supplier to OEM" use case defines the exchange of JT files to the OEM.

Common to both sections is that the use cases provide a basis for further use cases. This means that by the Supplier or the OEM more specific use cases (viewing, design in context, etc.) will be applied subsequently. The most important requirements are:

- Quality check of JT and validation against native CAD model.

- Meta data must be contained in JT model.

- Removal of intellectual property (if necessary).

\section{$4 \quad$ Evaluation and Testing}

An important aspect in driving the application and development of JT is a coordinated approach between the different bodies on the one hand and a coordinated approach of the activities themselves on the other hand. 


\subsection{Involved Bodies}

Basis for the standardization activities of JT were the enormous efforts of many international bodies. After the initial impact has be accomplished by SASIG [1], the four bodies ProSTEP iViP Association (PSI), the German Association of the Automotive Industry (VDA), the Automotive CIO's and the Global Automotive Advisory Group for PLM (GAAG) have played a leading role (Figure 5). Therewith ProSTEP iViP (www.prostep.org) has driven the activities thoroughly.

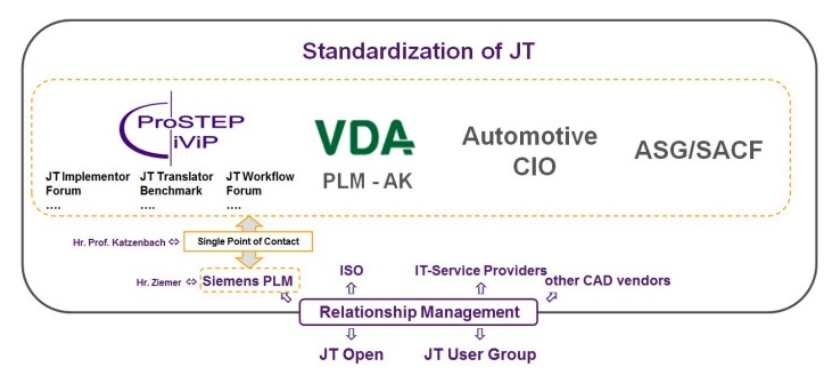

Fig. 5. International cooperation in the standardization of JT

Last but not least the MoU (Memorandum of Understanding) between Siemens PLM und ProSTEP iViP provides the basis between both parties in the area of JT, in order to foster a common understanding of goals and measures to establish JT as binding process format.

\subsection{National and International Coordination}

In 2009 the ProSTEP iViP Association, in a joint effort with the VDA (German Automotive Association), has started three different but interacting world-wide unique JT activities. Within the so-called JT Workflow Forum Use Cases for the application of JT are specified, describing the common view from industry. The JT Implementor Forum provides support in JT translator development maintains a platform for interoperability testing for the vendors. Within the JT Application Benchmark the requirements are comprehensively tested within neutral environment.

The project plans of these three project groups are well-harmonized, for driving the application of JT as a common effort.

JT Workflow Forum: Aim of the project is to define and prioritize key use cases and to identify relevant downstream processes for the JT based data exchange. For all use cases requirements are collected and test criteria defined e.g. under the aspects of exchanging of visualization data, the validation of 3D geometry, GD\&T (Geometric Dimensions and Tolerances) and last but not least translator quality.

These tasks are supported by the Content Harmonization subgroup, which is responsible for providing a clear definition of which requirements should be satisfied by the JT format and which by STEP AP242 as backbone format. 
JT Implementor Forum: Within the framework of the JT Implementor Forum Project Group, a platform for interoperability testing for the vendors is maintained. It is a forum where participating vendors can test the translators that they are developing "behind closed doors". The JT Implementor Forum is a neutral forum for software vendors, where they can perform tests in an atmosphere of mutual trust and exchange information on experience already gained.

In addition, from the vendor perspective, the activities within the JT Implementor Forum can be seen as a kind of preparation for the JT Application Benchmark.

JT Application Benchmark: Based on the recent standardization activities of JT and also STEP AP 242, it is of importance to assure data exchange quality within a neutral Benchmark and to provide support in JT translator development.

The ProSTEP iViP Association, together with the VDA PLM working group, has initiated a first JT translator benchmark in 2009. Subsequent the second translator benchmark was conducted in 2010. The third benchmark was completed by the end of 2012. Thereby the focus will be laid on three main topics:

1. CAD to JT export, focus on LOD and PMI.

2. JT viewing, focus on performance and functionality

3. Assembly conversion with STEP AP 242 XML and JT

Vendors participating in the benchmark are invited to present their latest functionalities in form of Showcases to the JT Workflow Forum members. In focus are cuttingedge solutions that demonstrate the possibilities of the JT format and applications. There are still small functional errors and therefore need for improvement (Figure 6).
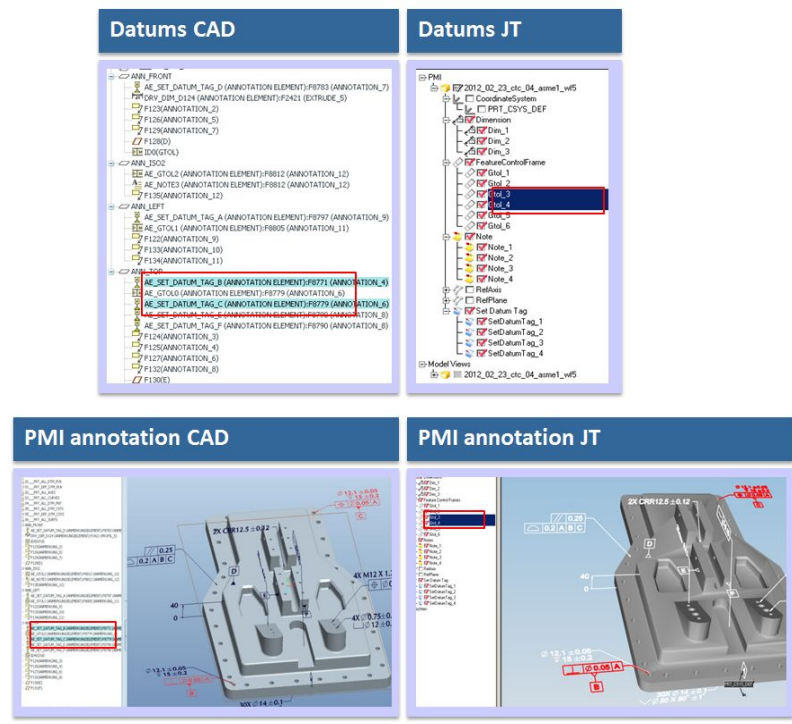

Fig. 6. Results of recent JT benchmark 


\section{$5 \quad$ Pilot Projects}

As a further step towards the productive usage of JT in automotive downstream processes, first pilot projects are established. Volkswagen decided to use JT for internal downstream processes to eliminate the need for drawings [7] [8] (Figure 7). This application is limited to the use case "single parts" and shall be subsequently extended to the full assemblies to avoid the usage of huge JT monolithic files.
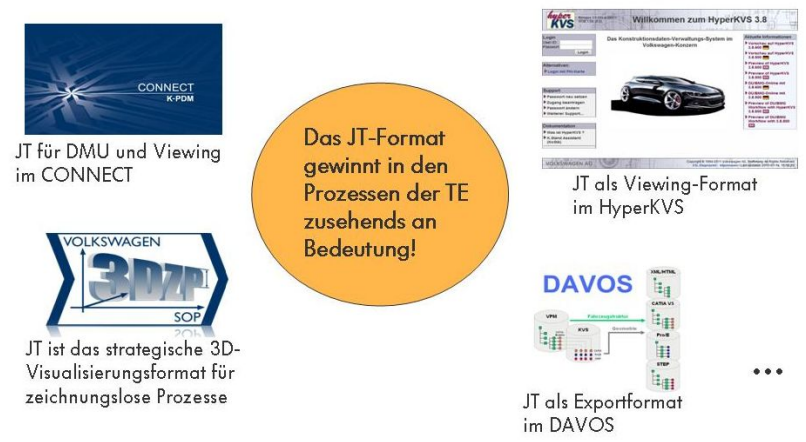

Fig. 7. Usage of JT within the Volkswagen Group

One of the most challenging project is the Daimler/Continental JT pilot project by exchanging an ESP Electronic Control Unit.

In the following the process landscape (Use Case: "Supplier to OEM") of the Daimler/Continental JT-pilot project is shown (Figure 8).

In order to fulfill the OEM needs (e. g. in case of data quality) it has to be ensured that the JT and the accompanying structure formats are created properly. In a first step the configuration settings of the JT-translator have to be adjusted to the OEM needs. Therefore the mentioned ProSTEP iViP JT Content Harmonization workgroup (JTCH) has created a first best practice document [9]. In the second step, the OEM specific data preparation has to be done. Therefore the Daimler JT Supplier Package (JTSP) is available for all Daimler suppliers [7].

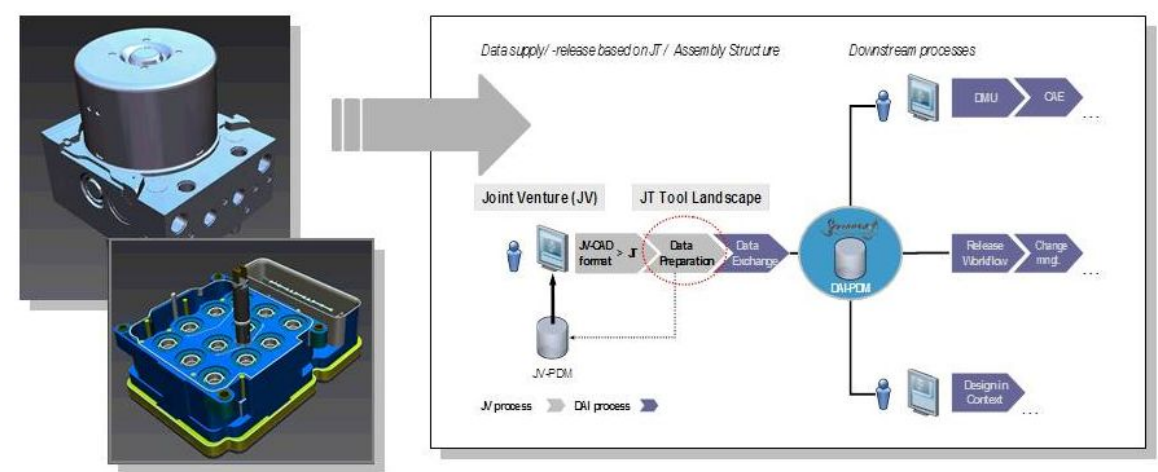

Fig. 8. Usage of JT in partner integration [7] 
The Daimler JTSP offers the opportunity to edit the JT internal attributes and to prepare XML based structure formats to meet the OEM requirements. The third step in the process landscape is the data exchange to the OEM. In case of the Daimler AG the OFTP based SWAN system is used.

The final step of this process is the data import into the Daimler PDM system Smaragd. At this point in time the externally created JT datasets are released and available to all Daimler downstream processes such as Viewing, DMU or Design in Context [10].

Meanwhile the full CAD data exchange by using JT is going to be adopted as base CAD translation technology by supplier portals like OpenDESC.com (www.opendesc.com) [11].

\section{Conclusions}

This contribution addresses the state of the art activities in in order to establish JT as an universal process format. Industry needs simple solutions for the efficient use of IT technology inclusive consistency in processes. There are strong requirements for lightweight 3D formats for the visualization and downstream processes, complementary formats in order to exchange meta data, structure data and kinematics data as well as open and standardized formats to reduce total cost of ownership and to minimize dependency of single vendors [8], [9], [10].

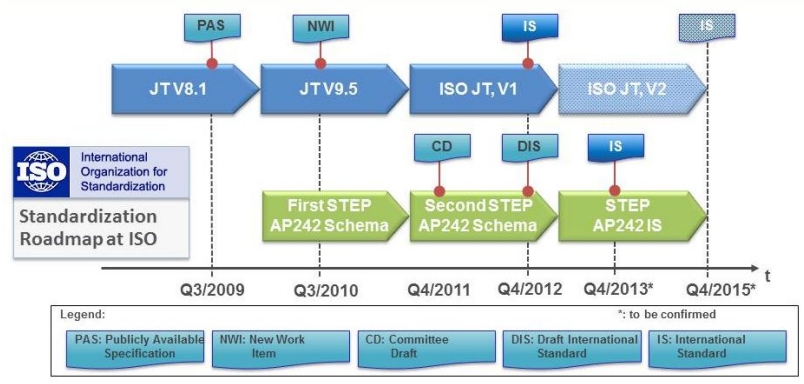

Fig. 9. The schedule of JT and STEP AP 242

After these very promising pilot projects mentioned above the activities on the subject of lightweight visualization based on JT have already reached a good level of maturity encouraging the wide range of companies and users, whereas it is a forthcoming task to push the integration with the accompanying format STEP AP242 (Figure 9). However first applications creating or using STEP AP242 XML as a outor input format are already available today, the wide usage will be announced in the next years. The future data exchange process will adopt JT as well as STEP AP242 ensuring the exchange of whole complex products in all stages of product and process development (Figure 10). 

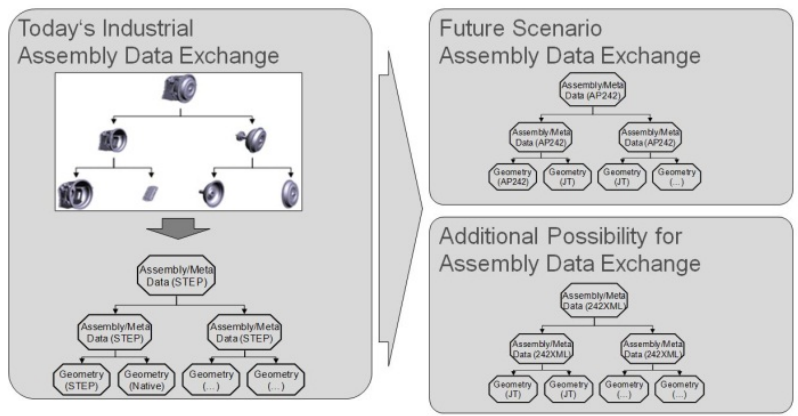

Fig. 10. Assembly data exchange with JT and STEP AP 242

\section{References}

1. N. N.: Digital Engineering Visualization, SASIG, SASIG Guideline (2005), http:// www. aiag.org/source/Orders/index. cfm? search=D-21

2. N. N.: Collaborative Product Visualisation - General issues and use case description, Frankfurt, VDA, VDA Empfehlung 4966 (2007), http://www.prostep.org/ fileadmin/freie_downloads/Empfehlungen-

Standards/VDA/VDA_4966_Collaborative-ProductVisualization_1.0.pdf

3. Fröhlich, A.: White Paper 3D Formats in the Field of Engineering - a Comparison, PROSTEP AG, Darmstadt, http://www.pdfgenerator3d.com/nc/en/ product/white-paper.html

4. Biahmou, A., Fröhlich, A., Stjepandic, J.: Universelle Formate für Visualisierung und Konvertierung in der Engineering-Kollaboration. In: International Conference GO 3D, Rostock (2009)

5. Handschuh, S.: Wertextrahierende Nutzung von offenen leichtgewichtigen Datenformaten in automobilen Kollaborations- und Entwicklungsprozessketten, Technische Universität Kaiserslautern, PhD thesis (2011)

6. Beckers, R., Fröhlich, A., Stjepandic, J.: Anwendung und Potenziale universeller Visualisierungsformate. In: 9. Paderborner Workshop "Augmented \& Virtual Reality in der Produktentstehung", Paderborn (2010), http://www.transmechatronic.de/ uploads/tx_vitramemberadmin/literature/Anwendung_und_ Potenziale_universeller_Visualisierungsformate.pdf

7. Handschuh, S., Dotzauer, R.: ProSTEP Symposium 2012: JT on the Path of Enlightenment - Report of the JT working groups, Hamburg (May 10, 2012)

8. Röhl, H., Fröhlich, A.: Einsatz des JT-Formates in der Digitalen Fabrik. VDI Kongress "DigitaleFabrik", Regensburg (2012)

9. ProSTEP iViP, Whitepaper Applying JT - Guidance for Using JT in Practice Version 2 (2010), http://www.prostep.org/de/mediathek/veroeffentlichungen /white-paper-studien.html

10. Handschuh, S., Dotzauer, R., Fröhlich, A.: Standardized formats for visualization - application and development of JT. In: 19th ISPE International Conference on Concurrent Engineering, Trier (2012)

11. Bondar, S., Potjewijd, L., Stjepandic, J.: Globalized OEM and Tier-1 Processes at SKF. In: 19th ISPE International Conference on Concurrent Engineering, Trier (2012) 